\title{
Solaris One - A Serious Game for Thermodyanmics
}

\section{Dr. Ying Tang, Rowan University}

Ying Tang received the B.S. and M.S. degrees from the Northeastern University, P. R. China, in 1996 and 1998, respectively, and Ph.D degree from New Jersey Institute of Technology, Newark, NJ, in 2001. She is currently a Professor of Electrical and Computer Engineering (ECE) at Rowan University, Glassboro, NJ. Her research interests include virtual reality and augmented reality, artificial intelligence, and modeling and scheduling of computer-integrated systems. Dr. Tang is very active in adapting and developing pedagogical methods and materials to enhance engineering education. Her most recent educational research includes the collaboration with Tennessee State University and local high schools to infuse cyberinfrastructure learning experience into the pre-engineering and technology-based classrooms, the collaboration with community colleges to develop interactive games in empowering students with engineering literacy and problem-solving, the integration of system-on-chip concepts across two year Engineering Science and four year ECE curricula, and the implementation of an educational innovation that demonstrates science and engineering principles using an aquarium. Her work has resulted in over 90 journal and conference papers and book chapters.

\section{Dr. Kauser Jahan, Rowan University}

Kauser Jahan completed her Ph.D. studies in the Department of Civil and Environmental Engineering at the University of Minnesota, Minneapolis in 1993. She holds a B.S. degree in civil engineering from the Bangladesh University of Engineering and Technology and an M.S.C.E. from the University of Arkansas, Fayetteville. After completion of her graduate studies, she worked as an environmental engineer for the Nevada Division of Environmental Protection (NDEP). Her research interests include bioremediation of contaminated groundwater and soils; the fate and transport of pollutants in the environment;biodegradation of industrial and municipal wastewaters; physicochemical treatment of water and wastewater treatment; applied microbiology in environmental engineering. She is also active in K-12 STEM initiatives.

\section{Dr. Sachin Shetty, Tennessee State University}

Sachin Shetty is currently an Assistant Professor in the Department of Electrical and Computer Engineering at Tennessee State University. He received his Ph.D. degree in Modeling and Simulation from Old Dominion University in 2007 under the supervision of Prof. Min Song. His research interests lie at the intersection of computer networking, network security and machine learning. Recently, he has been working on security issues in cloud computing, cognitive radio networks, and wireless sensor networks. Over the years, he has secured funding over $\$ 3$ million from NSF, AFOSR, DOE, DHS, TBR and local industry for research and educational innovations. He has authored and coauthored over 30 technical refereed and non-refereed papers in various conferences, international journal articles, book chapters in research and pedagogical techniques. He is the director of the Cyber Defense and Security Visualization Laboratory (http://cyberviz.tnstate.edu/)

\section{Mr. Christopher Joseph Franzwa}




\title{
Solaris One - A Serious Game for Thermodynamics
}

\begin{abstract}
Stagnating growth in our educational systems has piqued interests in alternative teaching methods such as the inclusion of "serious games" into curricula. In response to those needs, a series of educational games have been developed in accordance with pre-engineering programs such as Project Lead the Way (PLTW). The focus of development is in creating an engaging, educational environment by balancing fun and learning whilst meeting the standards of commercial-level games and engineering and science curricula. This paper, in particular, presents the design and pedagogical methods, and the implementation of those methods, in a thermodynamics serious game, Solaris One, that accommodates the integration of game mechanics with learning. The game has broad learning outcomes, namely enhanced understanding of thermodynamics laws, strengthened problem-solving skills, and increased knowledge and skills of using games, simulation, modeling and collaborative learning tools.
\end{abstract}

\section{INTRODUCTION}

Amongst the complex educational development cycle, high school serves as the most defining point for growth and decision that ultimately affects a student's choice for career path. Unfortunately, difficulty with early concepts in mathematics and sciences can dissuade students from pursuing further education in STEM fields (Science, Technology, Engineering, and Medicine) [1]. Student frustration is commonly to blame for poor performance, but the wide range of learning styles and varying degrees of propensity between students makes it difficult for classic teaching methods to be universally effective. Even in modest class sizes, short class times do not allow instructors to provide individualized assistance given curriculum requirements.

Taking advantage of the tremendous revolution that interactive technologies have brought to consumers, serious games offer a number of strong learning-enhancement capabilities, allowing for the realization of virtual worlds that can adapt to and assist students in ways that the typical classroom environment cannot [2]. In standard textbook-driven lecturing and study, visual or hands-on learners are left to find their own ways of perceiving the ever more complex concepts as they wade through a course. Currently, even hands-on approaches to learning, such as lab experiments, are limited by budgetary and safety constraints. Virtual environments allow infinite potential for scientific exploration, offering scenarios that are simply impossible in reality.

In an effort to meet the educational needs of students and supply tools for instructors, a series of serious games under the umbrella theme of a "Sustainable City" have been produced [3]. Not seeking to replace or interrupt current curriculums, each game serves as a replacement for a lab, providing a similar exploratory experience. Solaris One follows a set of generic goals for any high-school oriented serious game, but here specifically we aim to enhance understanding of basic thermodynamic laws intuitively as well as mathematically. Textbooks have always provided example-based questions, but the student can be lost in wording. Here we present exactly what those textbooks intend, but in a more interactive, visualized way. Although the common assumption is that today's students are well integrated with electronics 
and games, we can't assume base knowledge of common software, thus it is desirable to place design emphasis on scalable difficulty and usability.

\section{Overview of Solaris One: Instructional goals}

Solaris One is the most recent entry in an overarching collaboration of games that seeks to create a consistent educationally-minded world that covers topics ranging from digital circuits to sustainable energy. In this world, the player takes the role of an engineer who is called to aid in a number of local city disasters. Sustain City, as the city is called, is an openworld environment that serves as a hub to each game, offering a backdrop for the player to navigate and explore. Each game, although unique and independent of the other, contributes to a singular concept of community enhancement through scientific pursuit.

The module presently in development, Solaris One, is a serious game that teaches thermodynamics. Thermodynamics can be a daunting topic due to its abstract concepts and unobservable phenomena. Rather than teach baseline thermodynamics, we seek to offer students the opportunity to apply learned knowledge to a series of mini-games that place one or multiple laws of thermodynamics into action. Although the examples can best be described as "real-world" applications, many of them have been simplified for the sake of appealing to a high school level audience; which usually do not have in-depth knowledge of calculus and other college-level mathematics.

Taking full advantage of the limitless possibilities virtual worlds offer, Solaris One finds the student leaving not only Sustain City, but the Earth completely as they travel to an asteroid floating above its atmosphere. The asteroid serves as a geo-synchronous power plant, absorbing pure solar radiation through extensive solar paneling and "beaming" it back to Earth via a parabolic dish. The adventure begins with an introductory mini-game that focuses on the most basic algebraic heat transfer equation. To escape from Earth's orbit, the player is given a rocket with a fuel system based on energy transferred through heat. Five cores are presented; each core representing energy stored in either the left or right thruster. The student must select the proper final temperature of the energy cores to facilitate the required energy transfer. To enforce critical thinking, the student is not asked to merely solve a singular equation to obtain an answer, but to solve a more complex optimization problem. The total temperature in degrees kelvin that each node can be raised by is capped, so it must be distributed such that each rocket obtains both high enough energy levels for lift off as well as a balance such that the rocket does not fly off course. Supplying the student with an equation and asking them to solve for a single variable fails to check for understanding. Such an exercise tests a student's understanding of algebra rather than of, in this case, thermodynamics. By presenting a scenario as shown in Figure 1 that is not a straight forward application, although not overwhelmingly difficult, the student must apply their understanding of the equations to find an answer. Each failure is met with a crash simulated based on the student's inputs and a success results in a safe transition to space as shown in Figure 2.

The following components of the game take place amongst an open region of the asteroid station known as Solaris One. Following a disastrous solar flare, two major systems on the space station become unstable; the parabolic dish beaming energy back to Earth as well as the energy distribution system running throughout the asteroid. To fix the two systems, there are two mini-games that the player must complete to accomplish their mission. Because the asteroid is open to exploration, the games do not have to be completed in any particular order. 


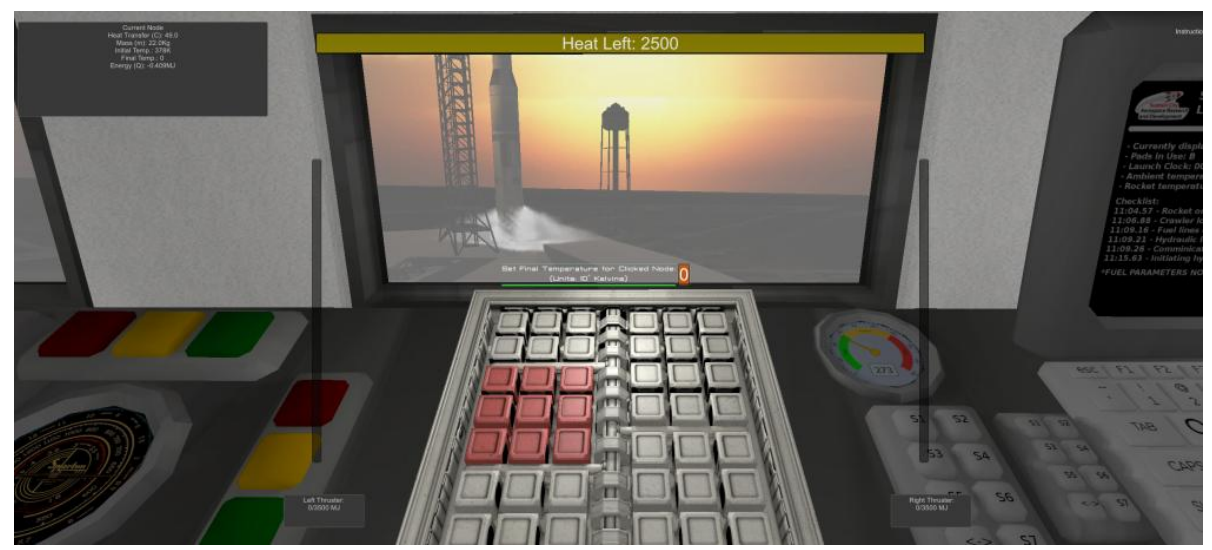

Figure 1: An image of the interface for the rocket launch game.
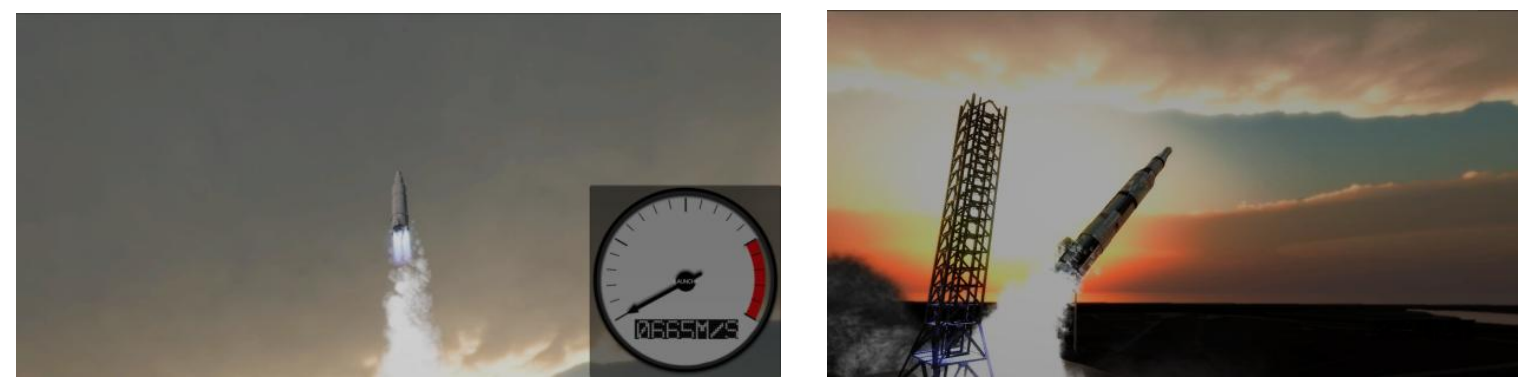

Figure 2: (a) A successful rocket launch (b) Rocket running off the track due to unbalanced energy production;

To repair the energy transmission dish, a large fuse array must be refitted with new fuses. Each fuse slot requires a fuse that suits its power rating. Each fuse is assigned random variables, asking the player to choose from fuses made of various materials, each with differing conductive properties. As was the case with the first mini-game, the student only requires knowledge of the algebraic heat transfer equation to solve the thermo equation at the heart of the problem. To enforce critical thinking and link thermodynamic concepts to practical situations, some variables are not directly supplied to the student, forcing them to obtain them from basic laws of electricity. The example relates electrical energy dissipation with thermodynamics laws. To discourage guess-and-check behavior, there are four fuse slots that must be calculated as shown in Figure 3.

To transmit energy throughout the asteroid, several piping fixtures must be repaired (Figure 4). The student enters a "digging" device that moves throughout the asteroid to a number of pipe sites for maintenance. Unlike the first two games, the pipe game serves as an application of the heat transfer equation to a new concept: heat loss and heat gain into a medium. As is the case in many real-world applications, heat loss or gain must be accounted for in piping systems. When transporting hot water to a restroom or kitchen, improper insulation can result in burns or, given long enough distances, the water can arrive cold. The student is presented with a series of small heat loss problems that grow in complexity as they continue through each level. Pipe paths and variables are generated procedurally to offer completely unique problems between players. 


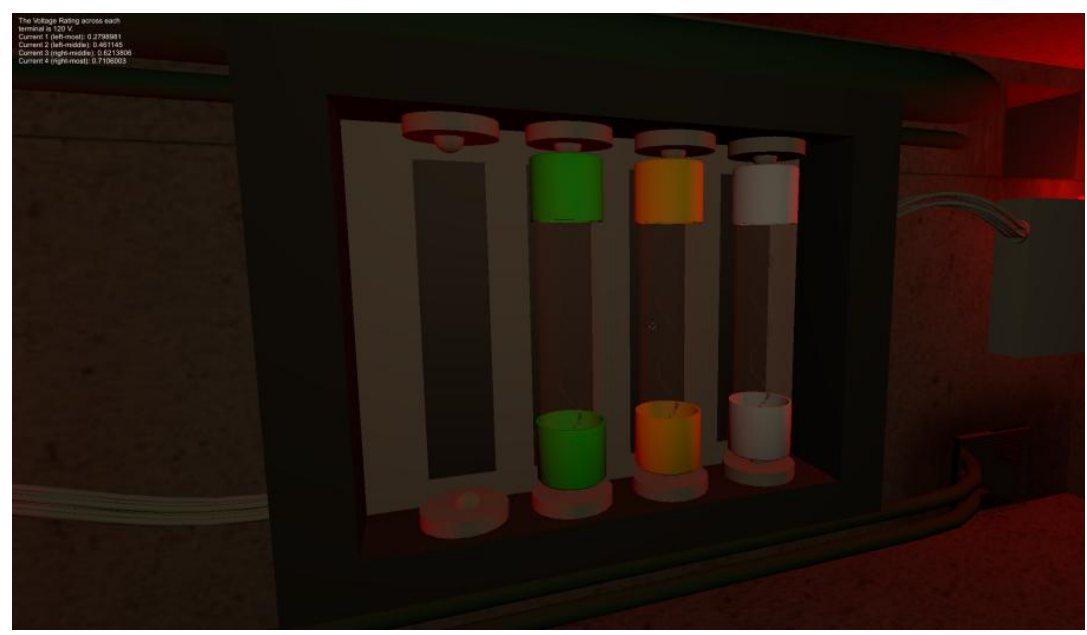

Figure 3: An image of the fuse array for Solaris One's fuse game.

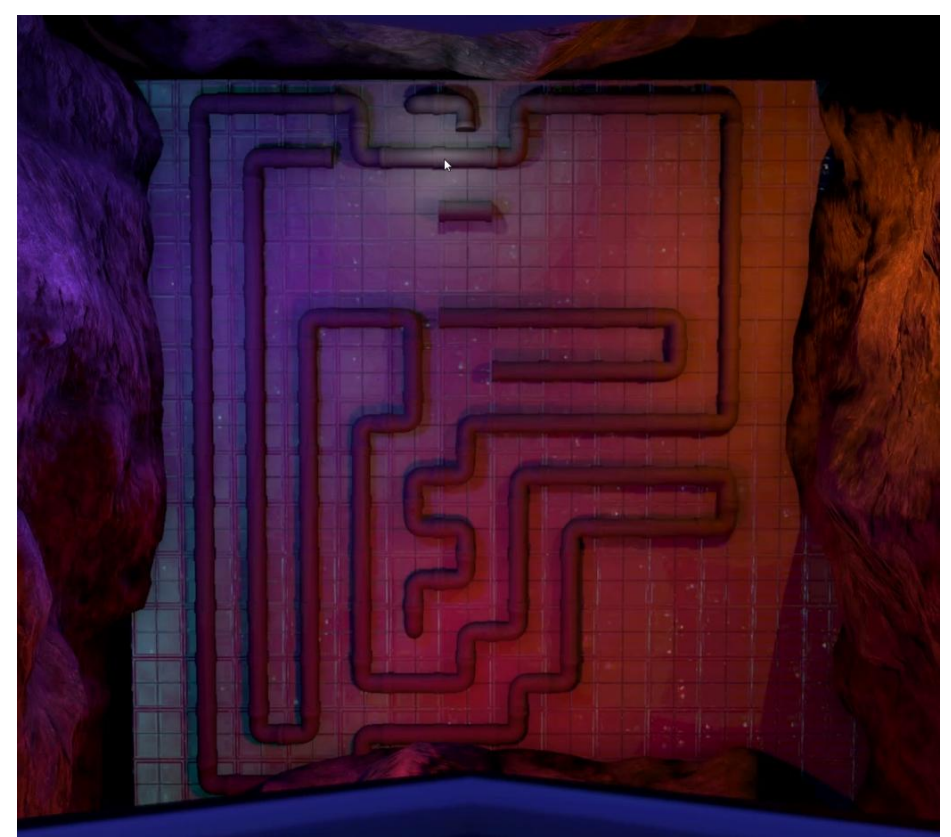

Figure 4: An image of the pipe game in Solaris One

\section{Overview of Solaris One: Design Focuses}

The action of transforming an educational topic into a virtual world is not enough to produce an effective learning tool. Concentrated effort must be placed on striking a balance between student interest and student education. A balance of learning and fun is an issue that has commonly been discussed within the realm of Serious Games [4], yet has not been explicitly solved. To develop an effective serious game, we have identified three essential design points that are taken into account for all games under the Sustain City project. These design points are:

1. The synthesis of a narrative with learning material to produce a logically progressive and engrossing story.

2. Supplementing player actions with either positive or negative feedback to encourage growth and reward high performance. 
3. Developing a sufficient guidance system to ensure that the student kept on track both in the game world and in the educational topics.

Ideally, a student is motivated to learn and develop on their own. Unfortunately, the vast majority of students are poorly motivated due to outside temptations, difficulties learning the material, or in some cases of laziness. To grasp and hold their attention, motivators must be fabricated that make learning fun or, to some degree, addictive. Educational material presented amongst STEM subjects tend to be administered in a dry, straight-forward way. To students that are not predisposed to the subjects, meaning is often lost in the vast oceans of theories and equations that are presented with little "real" examples to relate them to.

When looking at successful game franchises with a dedicated and sizable fan base, there are many examples of games that rely entirely on narrative. Without a strong narrative, games such as Mass Effect, Final Fantasy, and other Roleplaying Games (RPGs) would lose their appeal and not be able hold player attention with their repetitive gameplay. The obvious conclusion to be drawn is that the narrative design of a game can, on its own, serve as enough incentive to drive a player forward. In the classroom, repetition is a common method of learning, by solving a problem over and over with slightly different variables or composition [5]. Although these same learning concepts are put to action in Solaris One, there is a sense of progression and purpose that is lacking in a textbook. A student's success lends to advance the adventure and edge toward accomplishing both small local goals and the overarching goal of repairing the space station. Each mini-game present in Solaris One provides a logical reason for its existence and fits into a logical flow of events.

Reward/Punishment systems have long been considered tools for learning. Although there tends to be little exercised in terms of "punishment," rewards can provide immense incentive to achieve greater performance. Such concepts have been present in video games since their inception, present in the form of "High Scores" or special unlockable components. Punishment naturally takes the form of "Game Over" screens or losing points. Caution should be taken, however, in these types of punishments within a serious Game. Rather than frustrating and overwhelming a student with negative feedback, it is usually best to supply additional help or withhold rewards.

In Solaris One there are no instances of punishing the player, however there is a reward system in place for those that excel. Throughout the game, for each mini-game, monetary rewards are offered depending on performance. Regardless, a flat fee is supplied to every player for completing each game; however bonus cash is rewarded to those who complete more difficult problems, complete problems faster, or have lower number of attempts before success. Without some outlet to spend the cash, it becomes somewhat meaningless. On the asteroid, the primary form of movement for the player is a rover that is initially provided with very little speed and no special features beyond surface movement. With the monetary bonuses, players can purchase upgrades or perks to improve their gaming experience. Upgrades can be performance based (such as extra speed, thrusters, or better brakes for the rover), aesthetic (such as changing the color of the rover or adding parts), or additional features (such as vertical boosts or hovering).

It is a challenge in any game to teach a player how to play and to guide them through the game world. When dealing with an education-based game, the challenge intensifies due to the inherent variations in student backgrounds (e.g., highly self-motivated students vs. the ones lack motivation and prior knowledge), making the choice of how to guide the student from 
the start to the end of the game without direct instructor interactions a complex problem. Guidance is essential when trying to avoid frustration and losing a player to incidental game mechanics. In a serious game, guidance can be broken down into two categories: navigational guidance and educational guidance.

Navigational guidance refers to assisting a player through the game world. Such a system in Solaris One is modeled after those commonly found in modern games. For example, by leveraging the functionality of pathfinding techniques, lines are rendered within the game world, pointing the player toward the objective regardless of their position; akin to games such as Bioshock Infinite and Dead Space.

Educational guidance refers to assisting the player with difficulties experienced when solving problems throughout the game. In Solaris One, as well as all Sustain City games, we seek to first assess the student's skill to ascertain the amount and type of help to provide. Before each game, a set of multiple-choice questions are presented to find out what the student knows and what they may need help with as exemplified in Figure 5. In this way, the student can be assigned both a ranked proficiency as well as knowledge of specific topics in which they struggle or excel. Testing and feedback from previous systems developed within Sustain City has shown that when presented with instructions or dry educational references, the student usually skips it and does not return to it at any point. To avoid this, a different strategy is taken with the presentation of the games. In the pipe mini-game, a problem is presented with extreme simplicity, requiring minimal understanding of the subject manner. The game progresses through multiple stages, building up small changes to the concepts on each stage until the full problem is presented. In this way, the student is presented the information in a structural, incremental way, not overwhelming them all at once. References are still available to the player if they choose to use them.

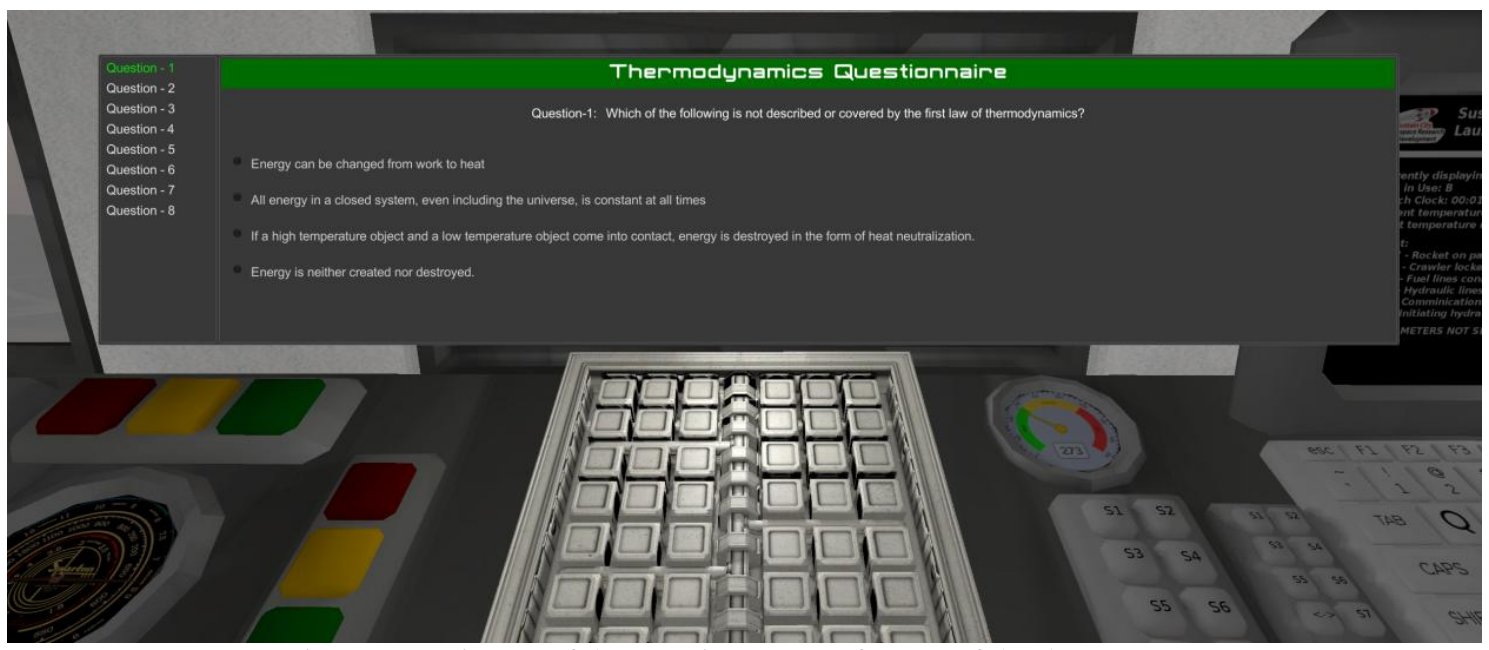

Figure 5: An image of the question prompt for one of the three games.

\section{Conclusion}

This paper presents an approach to serious games design within an example game (Solaris One) that teaches basic thermodynamics concepts. Solaris One is the culmination of many game development cycles and set to provide out most successful deployment yet. The learning materials provide an attractive and engaging environment for students to draw out their understanding of engineering texts, transform factual information into usable 
knowledge, and consolidate their perceptions of information through integrated real-life design applications.

\section{ACKNOWLEDGMENT}

This work is supported in part by the National Science Foundation grant \#OCI-1041306 .

\section{BIBLIOGRAPHY}

[1]. Douglas, J., Iversen, E., and Kalyandurg, C., "Engineering in the K-12 Classroom - An Analysis of Current Practice and Guidelines for the Future" http://www.asee.org/documents/conferences/k12/WorkshopDocuments/Engineering_in_t he_K-12_Classroom.pdf

[2]. Ying Tang, Sachin Shetty, Kauser Jahan, John Henry, and S. Keith Hargrove, "Sustain City - A Cyberinfrastructure-Enabled Game System for Science and Engineering Design," Journal of Computational Science Education, Vol. 3, No. 1, 2012, pp. 57-6

[3]. Chris Franzwa, Ying Tang, Aaron Johnson, "Serious Game Design: Motivating Students through a Balance of Fun and Learning," Proceedings of the $5^{\text {th }}$ International Conference on Games and Virtual Worlds for Serious Applications, Sept. 11-13, Bournemouth, UK, 2013, pp. 1-7

[4]. de Freitas, S. "Learning in Immersive worlds: A review of game-based learning"

[5]. J. M. Thomas and R. M. Young, "Annie: automated generation of adaptive learner guidance for fun serious games," IEEE Transactions on Learning Technologies, Vol. 3, No. 4, 2010, pp. 329-343 